\title{
Verification of Balloon Integrity by Ultrasound Imaging in Accelerated Partial Breast Irradiation
}

\author{
James D Christensen ${ }^{1}$, Mark G Trombetta ${ }^{1} \&$ Olivier Gayou ${ }^{1}$ \\ ${ }^{1}$ Department of Radiation Oncology, Allegheny General Hospital, United States \\ Correspondence: James D Christensen, Department of Radiation Oncology, Allegheny General Hospital, United \\ States. E-mail: james.christensen@ahn.org
}

Received: July 26, 2016

doi:10.5539/cco.v5n2p60

\author{
Accepted: August 15, $2016 \quad$ Online Published: October 27, 2016 \\ URL: http://dx.doi.org/10.5539/cco.v5n2p60
}

\begin{abstract}
Purpose: An overview of the ultrasound imaging artifacts encountered in Mammosite balloon APBI is presented. Analysis of ultrasound measurement errors and the dosimetric impact of balloon leakage were used to determine whether clinically relevant changes in balloon size can be reliably detected. Methods: Ultrasound imaging of a Mammosite balloon phantom was performed to better understand measurement errors and accuracy. The dose to the prescription point as a function of balloon diameter was computed for different sized balloons. The results were compared to phantom measurements of balloon diameter versus filling volume to assess the dose change that would result from tissue moving inward with a shrinking balloon boundary. In APBI patients undergoing a course of 10 treatment fractions, the accuracy and variability of balloon size measured with ultrasound imaging was compared to CT. Results: Ultrasound artifacts combine to form a false image of the distal balloon boundary. Proper US probe orientation and choice of measurement point locations improved distance measurement accuracy. A $1 \mathrm{~mm}$ change in balloon diameter is measurable with $\pm 0.1 \mathrm{~mm}$ error and corresponds to $<4 \%$ change in dose $1 \mathrm{~cm}$ from the balloon. Measurement errors relative to CT averaged less than $1.4 \mathrm{~mm}$ and variability (standard deviation) over the course of treatment averaged $1.9 \mathrm{~mm}$. Conclusions: Properly performed ultrasound image acquisition and analysis can detect dosimetrically relevant changes in the size of a leaking balloon. This study confirms that US imaging is a valid method of verifying APBI balloon integrity over the course of treatment.
\end{abstract}

Keywords: breast cancer, partial breast irradiation, balloon, HDR, ultrasound, Mammosite

\section{Introduction}

\section{Background \& Prior Clinical Studies}

Accelerated partial breast irradiation (APBI) high dose-rate brachytherapy (HDR) is designed to treat post-lumpectomy breast cancer patients by sterilizing the post-surgical site of microscopic tumor cells leading to reduced recurrence rates. The use of APBI is growing due to excellent outcomes, limited toxicity and positive patient satisfaction (Keisch et. al., 2003; Polgar et. al., 2004; Chen et. al., 2006). In five major trials with at least five years of follow-up, two of which have published 10 year data (Niehoff et. al., 2006; Hattangadi et. al., 2011; Shah, Vicini, Wazer, Arthur \& Patel, 2013), APBI rivaled traditional radiation treatment modalities in clinical outcomes while minimizing dose to normal tissues including the skin with resulting reduced side effects and improved cosmesis.

APBI brachytherapy uses a balloon inserted into a post-lumpectomy cavity and inflated with water to completely fill the cavity. The balloon has either one or multiple catheters (4 in the Mammosite Multi Lumen device), which run through the balloon and enable an Ir-192 source affixed to a wire to be positioned precisely within the balloon. A remote after-loader unit moves the source in steps through multiple positions calculated by the planning system to optimize the dose distribution. Rapid dose fall-off from the edge of the target volume is achieved due to the steep inverse relationship of dose versus distance from the balloon. This enables dose sparing of distal organs at risk like the skin.

\subsection{Outline of the Problem}

The rapid dose fall-off also means that any change in balloon size, for example due to leakage of the fluid used to inflate the balloon at implantation, may lead to large changes in dose to the target and nearby normal tissues. 
One study reported balloon failure (rupture rate or leakage) in 5 of 8 implanted balloon catheters (Harper, Jenrette, Vanek, Aguero \& Gillanders, 2005), which was later attributed to a faulty batch. Larger clinical studies reported failure rates of 3\%-13\% (Keisch et. al., 2003; Niehoff et. al., 2006; Hattangadi et. al., 2011; Shah et. al., 2013; Harper et. al., 2005; Forszpaniak, 2004).

The treatment target is generally breast tissue $1 \mathrm{~cm}$ from the balloon surface excluding skin, ribs and chest wall. A simple inverse square calculation predicts that for every $1 \%$ reduction from full balloon diameter the dose to the prescription point ( $1 \mathrm{~cm}$ from the balloon surface) will increase by about $1.5 \%$. For a typical $4 \mathrm{~cm}$ balloon, this translates to greater than $10 \%$ increase in dose for a $3 \mathrm{~mm}$ reduction in diameter. This assumes fully elastic breast tissue; for fully inelastic breast tissue, the source locations will shift from the cavity center due to gravity. A shrinking balloon's effects on real breast tissue could show both effects depending on the amount of filling fluid that is lost. Dose to organs at risk such as the skin also will change. A reduction in balloon diameter could result in large increases in dose to OARs and normal breast tissue outside the target volume. Clearly, a leaking balloon would impact the dose delivered to both target tissue and organs at risk.

Verification of the balloon's size over the typically week-long course of treatment is a key quality control measure that should be performed daily prior to each treatment (Shah, Wobb, Manyam, Khan \& Vicini, 2016). Some clinics use daily computed tomography (CT) scans to check balloon integrity (Keisch et. al., 2003; Esthappan et. al., 2009). However, recommendations for the minimization of imaging dose in radiotherapy (Murphy et. al., 2007), especially in younger patients, highlights the value of a non-ionizing imaging modality for repeated daily verification. Ultrasound has been used extensively for breast imaging given its excellent breast contrast, high spatial resolution and non-ionizing nature, and it is currently used by many clinics for daily verification of APBI balloon integrity. However, ultrasound imaging is prone to many types of image artifacts due to the reflection, refraction and attenuation of sound waves and these are well documented, especially for implanted objects with sharp contrast boundaries (Sites et. al., 2010; Perkins \& Price, 1983).

\subsection{Solutions to the Problem}

The APBI balloon applicator's specific structure leads to unique image artifacts that can obscure visualization of the balloon and interfere with measurement of its size. It is important to understand the basis of these artifacts so they can be recognized clinically and avoided to ensure the accuracy of balloon measurements. In a recent literature search, no published studies were found describing the particular artifacts encountered in balloon APBI or their impact on the monitoring of balloon size. Presented here is an overview of the ultrasound imaging artifacts encountered in MammoSite ${ }^{\circledR}$ balloon APBI together with methods to avoid these artifacts and obtain a simple yet robust metric to verify balloon integrity. Analysis of ultrasound measurement error and the dosimetric impact of balloon leakage were used to determine whether clinically relevant changes in balloon size can be reliably detected by US imaging.

\section{Methods and Materials}

\subsection{Patient Workflow}

Our institution is part of a clinical study by the National Surgical Adjuvant Breast and Bowel Project (NSABP B-39) and the Radiation Therapy Oncology Group 0413, which is exploring the use of APBI high dose-rate brachytherapy to treat post-lumpectomy breast cancer patients. Post-surgical implantation of a balloon applicator is performed with the size chosen to best fit the post-resection cavity $(3-6 \mathrm{~cm}$ diameter). The HDR prescription is 34 Gy delivered in 10 fractions treated twice per day over 5 days. The planning target volume is to points $1 \mathrm{~cm}$ distance from the balloon surface, excluding a $0.5 \mathrm{~cm}$ subcutaneous region that would be coexistent within the target volume and any tissue deep to the chest wall. The balloon/catheter applicator employed was the MammoSite ${ }^{\circledR}$ Multi-Lumen (MML) applicator shown in Figure 1. Varian treatment planning system Brachyvision $^{\circledR}$ (Varian Oncology Systems, Milpitas, CA) and the Varisource ${ }^{\circledR}$ Ir-192 remote after-loader system (Varian Oncology Systems, Milpitas, Ca) were used for HDR treatment. For planning simulation, the patients were imaged by CT using a Siemens Sensation ${ }^{\circledR}$ Open scanner (Siemens Corporation, Compton, CA) with axial slices. Figure 2 shows a CT image of a MML applicator implanted in a post-surgical lumpectomy cavity.

The diameter of the HDR balloon applicator was measured in a plane bisecting the balloon and perpendicular to the long axis of the applicator (axial with respect to the balloon). The diameter is measured in $\mathrm{x}$ and $\mathrm{y}$ directions within this plane to account for possible balloon asymmetry. The balloon diameter measurements are confirmed via ultrasound imaging using a Siemens Acuson X300 PE ${ }^{\circledR}$ (Siemens Corporation, Compton, CA) ultrasound device. This is done immediately following CT with the patient lying in the treatment position on the CT simulator table. At the time of subsequent treatments, the ultrasound measurements are repeated in the HDR treatment room to ensure that the balloon is intact and its diameter has not changed. 

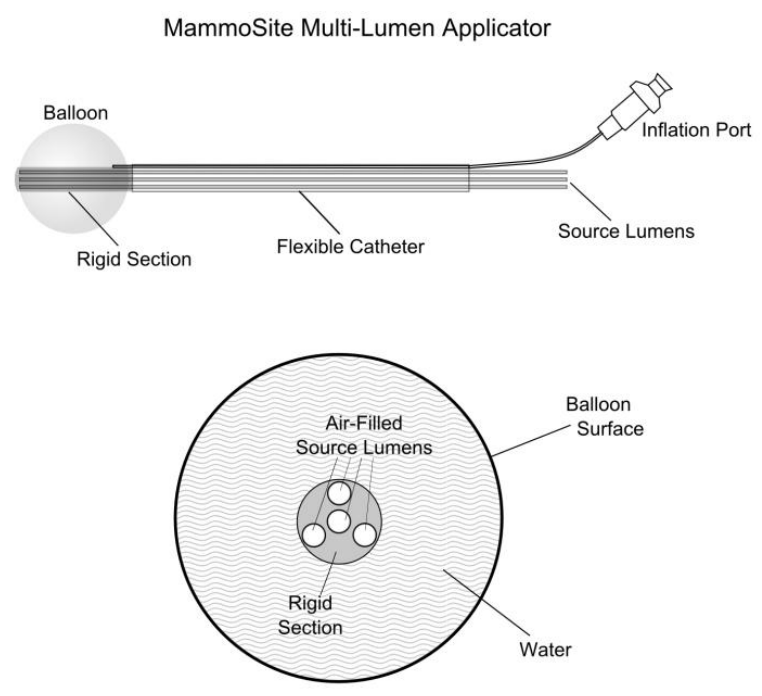

Cross-Section through Balloon Center

Figure 1. A diagram of the MammoSite ${ }^{\circledR}$ Multi-Lumen HDR balloon applicator

Top: Side view; Bottom: Cross-sectional view through the balloon showing the internal structure including the 4 source lumens.

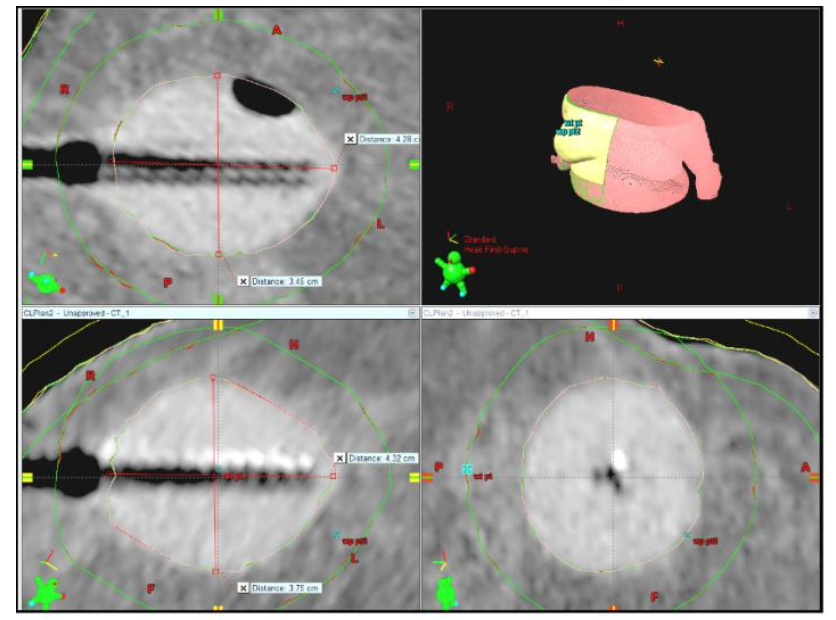

Figure 2. A simulation CT of a balloon applicator implanted in the right breast of an APBI patient is shown in the HDR brachytherapy planning software. Slice orientations are illustrated by the avatar in the lower left corner of each frame. The applicator has 4 catheters with a wire inserted into catheter \#1 for indexing identification

\subsection{Mammosite HDR Applicator}

The Mammosite HDR is shown in Figure 1. The device consists of a tube, the catheter, which is approximately $10 \mathrm{~mm}$ in diameter and connects to a balloon. 5 smaller tubes run through the catheter and into the balloon. One of these is used to fill the balloon with water after insertion and empty it before removal. There a different sized balloon which have diameters ranging from $3 \mathrm{~cm}$ to $5 \mathrm{~cm}$; this size is determined by the patient's lumpectomy cavity size. The other 4 tubes running through the applicator, the lumen, do not communicate with the inside of the balloon. They are air filled and each is connected by tubing to a different channel input on the HDR remote after-loader unit. These lumens are approximately $10 \mathrm{~mm}$ in diameter, are made of hard plastic and are air filled, even when the balloon is full.

\subsection{Phantom Imaging}

Ultrasound imaging of a phantom was performed to better understand US imaging artifacts created by the MammoSite ${ }^{\circledR}$ balloon and test the accuracy and precision of balloon measurements in a controlled environment. The phantom consisted of plastic tub filled with blended tofu into which a $4 \mathrm{~cm}$ MML balloon applicator was fully submersed (Figure 3). The ultrasound attenuation properties of the tofu provided image contrast between 
the inside and outside of the water filled balloon. Blending the tofu allowed it to fill completely the space surrounding the balloon as its size changed, similar to the post-mastectomy cavity in an elastic breast. A syringe connected to the water filling catheter provided control of the balloon fill volume. Measurements of the balloon diameter were taken using CT and ultrasound as the balloon volume was decreased in $1 \mathrm{cc}$ increments.

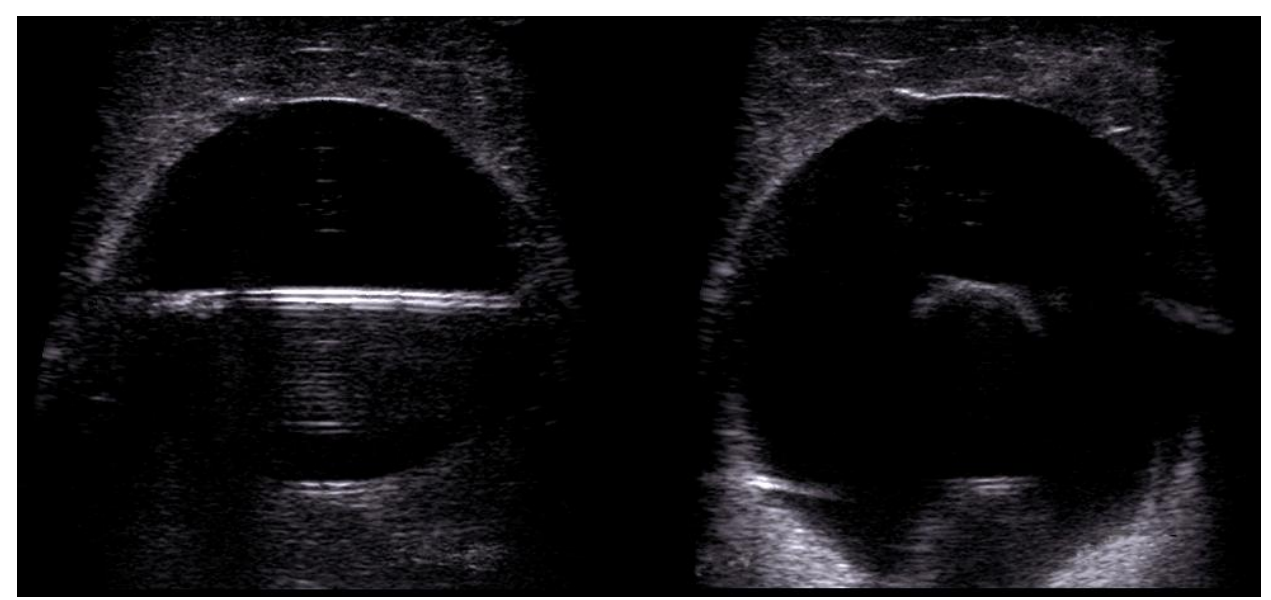

Figure 3. Ultrasound imaging of a tofu phantom was used to find the optimal probe orientation and distance metric for verifying balloon integrity. Left: The image plane is parallel to the long axis of the balloon applicator. Note the artifacts caused by signal reflection including the false distal balloon boundary. Measurement of the distance between the proximal balloon boundary and the proximal catheter is the only reliable measurement in this orientation. Right: the image plane is oriented perpendicular to the balloon axis. The cross section of the proximal edge of the internal catheters can be visualized; this strongly attenuating boundary causes the loss of signal from everything distal to it. The balloon border is well visualized at all other points in the image. Measurements of balloon diameter taken in this orientation correlate well with those obtained from CT images

\subsection{Dosimetric Effects of a Shrinking Balloon}

The dose to the prescription point $(1 \mathrm{~cm}$ distal to the balloon surface) as a function of balloon diameter was computed for different sized balloons. The dose calculation modeled a point source with inverse square dose fall-off and equal dwell times distributed evenly along all catheters. Comparison with dose distributions in patient treatment plans calculated by the planning system according to AAPM TG-43 (Nath et. al., 1995; Rivard et. al., 2004) showed excellent agreement.

The results were compared to dose distributions in patient treatment plans to assess the dose change that would result from tissue moving inward with a shrinking balloon boundary. These calculations were performed for different sized balloons, since a smaller balloon will have a larger dose change per $\mathrm{mm}$ change in diameter due to approximate inverse square fall-off from the source. The calculation point was $1 \mathrm{~cm}$ away from the balloon surface at a point perpendicular to the long axis of the applicator and midway along the balloon.

\section{Results}

\subsection{Ultrasound Artifacts}

Figure 4 shows an ultrasound image of an implanted MML balloon together with a diagram of the balloon structure and the position of image artifacts on the US images. Several image artifacts are present: 1) Reverberation artifact. This is caused by initial catheter edge. This is a well-known US artifact that results from sound waves making multiple reflections inside the catheter before traveling back to the probe. 2) Attenuation shadowing: The strongly reflecting air-water boundary at the proximal catheter edge together with the reverberation within the catheter described above causes a loss in sound wave amplitude seen as an image signal loss distal to the initial boundary. This leads to a loss of signal from the more distal catheters and a loss of signal of the distal balloon boundary. 3) A mirror artifact of the balloon curved surface caused by reflection between the proximal catheter and the proximal balloon boundary. The reflected sound waves travel twice the distance between these structures and the resulting time delay is interpreted by the US software as a signal originating at twice the deep, which generates a mirror image of the proximal balloon boundary. 

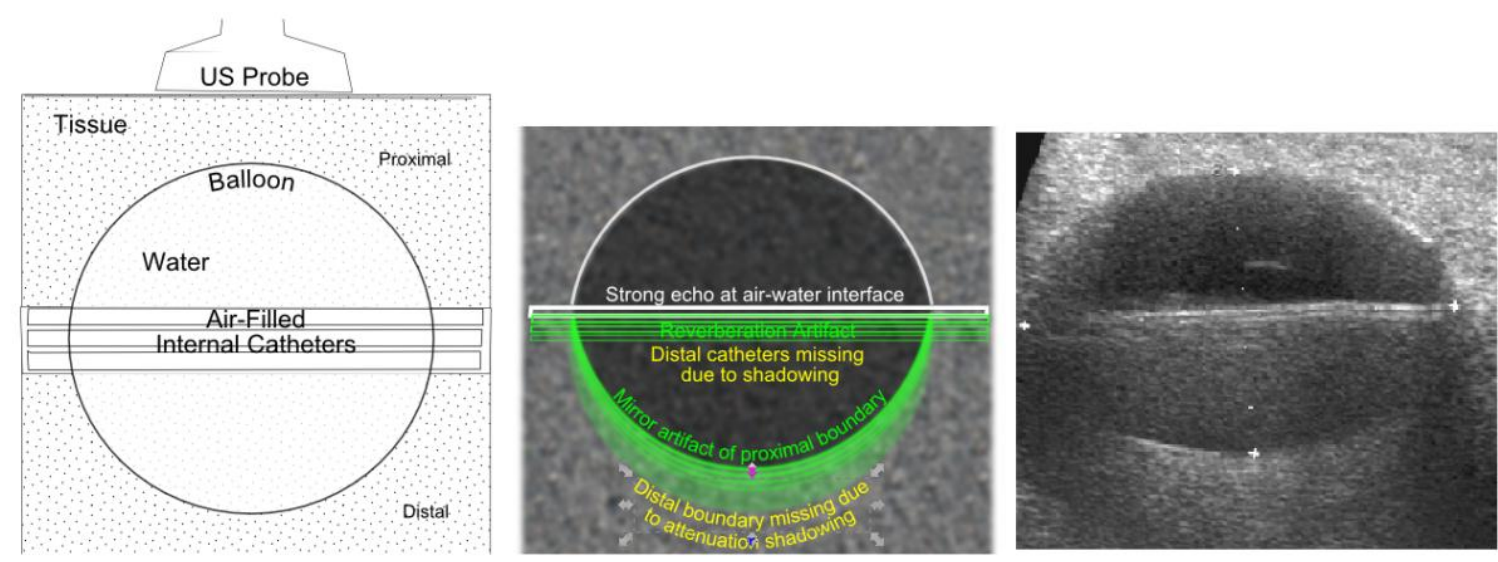

Figure 4. Left: A diagram of the the MammoSite ${ }^{\circledR}$ Multi-Lumen balloon applicator. Center: A schematic showing several US artifacts (green) produced by the applicator. The air-filled catheter most proximal to the US probe produces a strongly reflected signal. A first reflection by this catheter followed by a reflection from the proximal balloon boundary then a second reflection from the catheter combine to produces the image structure

labeled "mirror artifact of proximal boundary". This mimics the true distal balloon boundary but is in the incorrect location. Reflections within the proximal catheter cause reverberation artifacts mimicking the deeper catheters. The proximal catheter strongly attenuates signal transmission to deeper structures, thereby obscuring the deeper catheters and the distal balloon boundary. The result is an image of a symmetric balloon, but the image portion distal to the proximal catheter is comprised almost entirely of artifacts. Right: A clinical US image of a MammoSite ${ }^{\circledR}$ balloon implanted in an APBI patient demonstrating the described artifacts

The last two artifacts described above combine to form a false mirror image of the proximal balloon boundary in the image region where the true distal balloon boundary is missing. This mirror image is shifted by the thickness of the lumen containing the catheter bundle, which is $10 \mathrm{~mm}$ for the MML. Its position is also quite variable depending on the angle that the beam strikes the curved surface of the proximal catheter lumen.

\subsection{Phantom Measurements of Balloon Diameter versus Fill Volume}

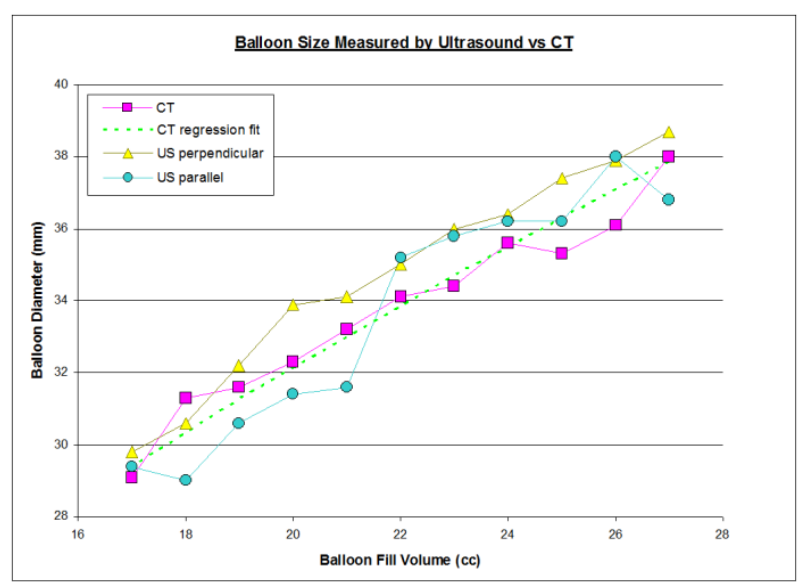

Figure 5. The balloon diameter was measured as a function of balloon filling. CT imaging served as the gold standard. Blended tofu was used to mimic breast tissue. Two different US measurement configurations were used: 1) With the US probe oriented perpendicular to the long axis of the balloon applicator, measurement of the

full balloon diameter was measured across a diagonal. 2) with US probe oriented parallel to the applicator, artifacts were avoided by measuring the shorter distance between the proximal balloon surface and the proximal internal catheter; the diameter was then calculated from known geometry $(2 *$ distance + constant). This measurement was more accurate, and was faster and easier to obtain

Figure 5 shows the CT and US measurements of balloon diameter as a function of balloon filling. Each cc removed from the balloon resulted in a reduction in balloon diameter of approximately $0.8 \mathrm{~mm}$. Of all methods used to measure balloon size, the ultrasound diameter measurement perpendicular to the applicator axis was the 
most precise, making it the best marker for balloon filling integrity. Diameter measurement became more difficult as the decrease in balloon diameter approached $10 \mathrm{~mm}$ due to buckling of the balloon surface, therefore results are shown up to $5 \mathrm{~mm}$ reduction. Within the balloon diameter range of $38-33 \mathrm{~mm}$, the diameter measured by US perpendicular to the catheter axis was fit by linear regression to the ideal model diameter calculated from the fill volume. The resulting slope was $0.93 \mathrm{~mm} / \mathrm{mm}$ with $\mathrm{r}^{2}=0.993$ and $95 \%$ confidence interval $0.84-1.02$. Therefore a $1 \mathrm{~mm}$ change in balloon diameter should be measurable with $95 \%$ confidence to less than $\pm 0.1 \mathrm{~mm}$ error. This $1 \mathrm{~mm}$ diameter reduction corresponds to a less than $4 \%$ change in dose at a distance $1 \mathrm{~cm}$ from the balloon.

\subsection{Measurement of Patient Balloon Diameter Error and Variability Over 10 Fractions}

In our patient population, the implanted balloon diameters ranged from 3.4 to $5.8 \mathrm{~cm}$. The median diameter was $4.0 \mathrm{~cm}$, and $95 \%$ were within 3.5 to $5.5 \mathrm{~cm}$. The absolute error in balloon diameter measurement by US imaging as compared to CT measurements and the variability in the US measurements over 1 week of treatment (10 fractions) were calculated for 4 patients. It was decided to use the measurement method with the US probe aligned parallel to the applicator axis. This ensured that the image plane bisected the balloon and the measurement was made through the balloon center. The catheter edge is clearly visible on captured images that are stored in the record and verify system for documentation of balloon verification and approval by the physician.

The diameter was measured from US images with the probe oriented parallel to the applicator through the balloon center, the distance from the proximal balloon boundary and the proximal edge of the catheter bundle; this distance was multiplied by 2 and added to the catheter bundle thickness to calculate the balloon diameter. The US measurement errors (relative to the CT diameter measured on the simulation day) averaged less than 1.4 $\mathrm{mm}$ and the variability (standard deviation) averaged $1.9 \mathrm{~mm}$.

\subsection{Dosimetric Effects of Changing Balloon Diameter}

Figure 6 shows the calculated dose to a point $1 \mathrm{~cm}$ from the balloon versus balloon diameter reduction. Comparison of the modeling results with actual patient plans showed good agreement. In our patient population, the mean implanted balloon diameter was $4.0 \mathrm{~cm}$ and $95 \%$ of patients had balloon diameters between $3.5 \mathrm{~cm}$ and $5.5 \mathrm{~cm}$. The results for these sized balloons are plotted in Figure 6. For a $2 \mathrm{~mm}$ reduction in diameter, the increase in prescription point dose was between 5-7\%. This is within the range of detectable balloon size change implied by the phantom results and about equal to the repeatability over multiple days measured in patients (Table 1).

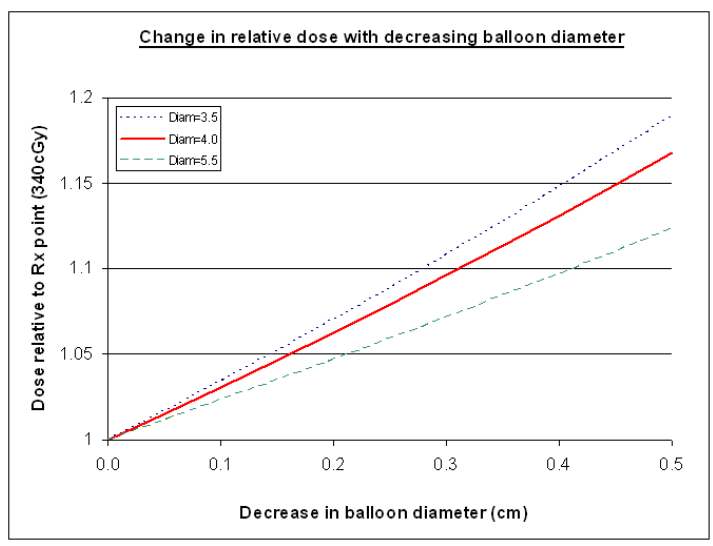

Figure 6. The change in dose to the prescription point $1 \mathrm{~cm}$ from the balloon as the balloon diameter decrease $\mathrm{d}$ from its full diameter.

Dose was modeled using the inverse square law with point sources distributed with equal dwell times along the 4 catheters. Comparison with patient treatment doses calculated in the planning system according to AAPM TG-43 showed excellent agreement. The median implanted balloon diameter for patients in our clinic was $4.0 \mathrm{~cm} ; 95 \%$ of implanted balloon diameters were between $3.5 \mathrm{~cm}$ and $5.5 \mathrm{~cm}$. The $2 \mathrm{~mm}$ accuracy of the ultrasound imaging methodology (Fig. 6 and Table 1) translates to approximately 7\% deviation from prescription dose 
Table 1. Analysis of Errors in Ultrasound Imaging Measurement of Balloon Diameter

\begin{tabular}{|c|c|c|c|c|c|}
\hline \multicolumn{3}{|c|}{ Measurement Protocol } & \multicolumn{4}{c|}{ Error (mm): } \\
Diameter(US)-Diameter(CT) \\
\hline $\begin{array}{c}\text { US Probe Orientation } \\
\text { vs Long Axis }\end{array}$ & Distance Measured & \multicolumn{2}{c|}{$\begin{array}{c}\text { Phantom } \\
\text { Mean }\end{array}$} & \multicolumn{2}{c|}{$\begin{array}{c}\text { Patients (n=12) } \\
\text { Mean }\end{array}$} \\
\hline$\perp$ & Balloon surface to proximal edge source lumen & -0.1 & 1.0 & -1.8 & 0.9 \\
\hline$\perp$ & Diagonal across balloon full diameter & 0.9 & 0.4 & -3.4 & 4.1 \\
\hline
\end{tabular}

* The distance measured with parallel probe orientation is doubled to calculate the balloon diameter (diameter $=$ $2 *$ distance + constant). Therefore for comparison the parallel mode standard deviation reported here is twice that of the actual measurement.

\section{Discussion}

Ultrasound imaging can detect the change in size of a leaking balloon that results in a roughly $10 \%$ increase in dose to the prescription point. Dosimetric changes to organs at risk such as skin would be greater or less than this depending on the tissue distance from the balloon surface. We decided to implement an action criterion of $2 \mathrm{~mm}$ for the distance measured by ultrasound each treatment day compared with the initial distance measured at simulation. If this criterion condition is not met, the patient has a repeat CT scan to compare with the initial CT balloon measurement.

Based upon our clinic's experience, we recommend verification testing of Mammosite balloon size using ultrasound imaging as part of the commissioning process prior to clinical implementation. It is essential to properly train staff that are tasked with the acquisition, interpretation and analysis of the US images. Complex and misleading image artifacts can be minimized by proper probe orientation with respect to the balloon applicator. Precise measurement of balloon size requires a careful choice of landmarks and consistent image analysis. Staff who will be performing the US imaging measurements should be trained in Mammosite applicator imaging, artifact formation and hands-on training with a phantom. For each patient, daily measurement of the balloon size should be performed with the patient in the treatment position immediately before treatment.

\section{Acknowledgements}

This work was described in part at the 2014 Annual Meeting of the AAPM, July 22, Austin, TX. Published abstract: Med. Phys. 41, 482 (2014).

\section{References}

Chen, P. Y., Vicini, F. A, Kestin, L. L., Wallace, M., Mitchell, C., Pettinga, J., \& Martinez, A. A. (2006). Long-term cosmetic results and toxicity after accelerated partial breast irradiation: A method of radiation delivery by interstitial brachytherapy for the treatment of early-stage breast carcinoma. Cancer, 106, 991-9. http://dx.doi.org/10.1002/cncr.21681

Esthappan, J., Santanam, L., Yang, D., Mutic, S., Low, D. A., \& Grigsby, P. W. (2009). Use of serial CT imaging for the quality assurance of MammoSite ${ }^{\circledR}$ therapy. Brachtherapy, 8, 379-384.

http://dx.doi.org/10.1016/j.brachy.2009.04.004

Forszpaniak, J. (2004). Balloon brachytherapy. Nowotwory J. Oncology, 54, 11-14.

Harper, J. L., Jenrette, J. M., Vanek, K. N., Aguero, E. G., \& Gillanders, W. E. (2005). Acute complications of MammoSite ${ }^{\circledR}$ brachytherapy: a single institution's initial clinical experience. Int. J. Rad. Onc. Biol. Phys., 61(1), 169-174. http://dx.doi.org/10.1016/j.ijrobp.2004.03.029

Hattangadi, J. A., Powell, S. N., MacDonald, S. M., Mauceri, T., Ancukiewicz, M., Freer, P., ... Taghian, A. G. (2011). Accelerated partial breast irradiation with low-dose-rate interstitial implant brachytherapy after wide local excision: 12-year outcomes from a prospective trial. Int. J. Rad. Onc. Biol. Phys, 83(3), 791-800. http://dx.doi.org/10.1016/j.ijrobp.2011.09.003

Keisch, M., Vicini, F., Kuske, R. R., Hebert, M., White, J., Quiet, C., ... Streeter, O. (2003). Initial clinical experience with the MammoSite ${ }^{\circledR}$ breast brachytherapy applicator in women with early-stage breast cancer treated with breast-conserving therapy. Int. J. Rad. Onc. Biol. Phys, 55(2), 289-293. http://dx.doi.org/10.1016/s0360-3016(02)04277-3

Murphy, M. J., Balter, J., Balter, S., BenComo, J. A., Das, I. J., Jiang, S. B., ... Yin, F. F. (2007). The management of imaging dose during image-guided radiotherapy: Report of the AAPM Task Group 75. Med. 
Phys, 34(10), 4041-4063. http://dx.doi.org/10.1118/1.2775667

Nath, R., Anderson, L. L., Luxton, G., Weaver, K. A., Williamson, J. F., \& Meigooni, A. S. (1995). Dosimetry of interstitial brachytherapy sources: Recommendations of the AAPM Radiation Therapy Committee Task Group No. 43. Med. Phys, 22, 209-234. http://dx.doi.org/10.1118/1.597458

Niehoff, P., Polgar, C., Ostertag, H., Major, T., Sulyok, Z., Kimmig, B., \& Kovacs, G. (2006). Clinical experience with the MammoSitew radiation therapy system for brachytherapy of breast cancer: Results from an international phase II trial. Radiation and Oncology, 79, 316-320. http://dx.doi.org/10.1016/j.radonc.2006.05.010

Perkins, A. C., \& Price, H. M. (1983). Catheter balloon artifacts in ultrasound imaging of the urinary bladder. $J$ Clin Ultrasound, 11, 110-113. http://dx.doi.org/10.1002/jcu.1870110213

Polgar, C., Major, T., Fodor, J., Nemeth, G., Orosz, Z., Sulyok, Z., ... Kasler, M. (2004). High-dose-rate brachytherapy alone versus whole breast radiotherapy with or without tumor bed boost after breast-conserving surgery: Seven-year results of a comparative study. Int J Radiat Oncol Biol Phys, 60, 1173-1181. http://dx.doi.org/10.1016/j.ijrobp.2004.05.012

Rivard, M. J., Coursey, B. M., DeWerd, L. A., Hanson, W. F., Huq, M. S., Ibbott, G. S., ... Williamson, J. F. (2004). Update of AAPM Task Group No. 43 Report: A revised AAPM protocol for brachytherapy dose calculations. Med. Phys, 31, 633-674. http://dx.doi.org/10.1118/1.1646040

Shah, C., Vicini, F., Wazer, D., Arthur, D., \& Patel, R. R. (2013). The American Brachytherapy Society consensus statement for accelerated partial breast irradiation. Brachytherapy, 12(4), 267-277. http://dx.doi.org/10.1016/j.brachy.2013.02.001

Shah, C., Wobb, J., Manyam, B., Khan, A., \& Vicini, F. (2016). Accelerated partial breast irradiation utilizing brachytherapy: patient selection and workflow. J. Contemporary Brachytherapy, 8(1), 90-94. http://dx.doi.org/10.5114/jcb.2016.58083

Sites, B. D., Brull, R., Chan, V. W. S., Spence, B. C., Gallagher, J., Beach, M. L., ... Hartman, G. S. (2010). Artifacts and pitfall errors associated with ultrasound-guided regional anesthesia. Regional Anesthesia \& Pain Med, 35(2), S81-S92. http://dx.doi.org/10.1097/aap.0b013e3181d3535a

\section{Copyrights}

Copyright for this article is retained by the author(s), with first publication rights granted to the journal.

This is an open-access article distributed under the terms and conditions of the Creative Commons Attribution license (http://creativecommons.org/licenses/by/4.0/). 